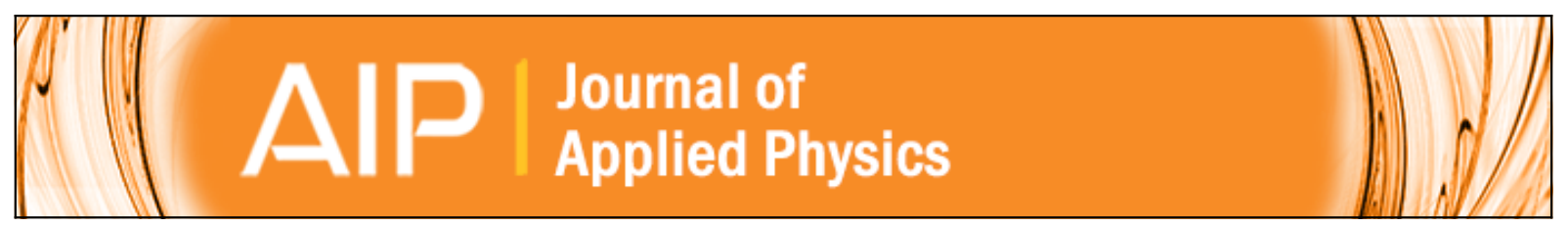

\title{
Structural and electrical properties of In-implanted Ge
}

R. Feng, F. Kremer, D. J. Sprouster, S. Mirzaei, S. Decoster, C. J. Glover, S. A. Medling, S. P. Russo, and M. C. Ridgway

Citation: Journal of Applied Physics 118, 165701 (2015); doi: 10.1063/1.4934200

View online: http://dx.doi.org/10.1063/1.4934200

View Table of Contents: http://scitation.aip.org/content/aip/journal/jap/118/16?ver=pdfcov

Published by the AIP Publishing

\section{Articles you may be interested in}

Direct observation of substitutional $\mathrm{Ga}$ after ion implantation in Ge by means of extended x-ray absorption fine structure

Appl. Phys. Lett. 101, 261904 (2012); 10.1063/1.4773185

Effects of temperature and near-substrate plasma density on the structural and electrical properties of dc sputtered germanium thin films

J. Vac. Sci. Technol. A 29, 051301 (2011); 10.1116/1.3607410

In-implanted ZnO: Controlled degenerate surface layer

J. Vac. Sci. Technol. B 27, 1593 (2009); 10.1116/1.3089375

Application of flash-assist rapid thermal processing subsequent to low-temperature furnace anneals J. Vac. Sci. Technol. B 24, 450 (2006); 10.1116/1.2140003

Structural and electrical properties of $\mathrm{p}+\mathrm{n}$ junctions in Si by low energy $\mathrm{Ga}+$ implantation J. Appl. Phys. 82, 4990 (1997); 10.1063/1.366367

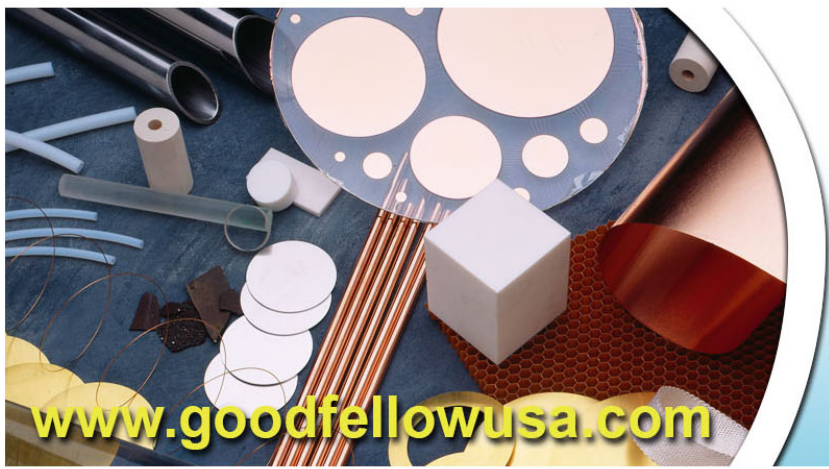

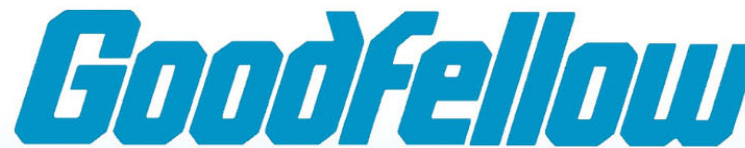

metals • ceramics • polymers composites • compounds • glasses

Save $5 \%$ B Buy online 70,000 products $\cdot$ Fast shipping 


\title{
Structural and electrical properties of In-implanted Ge
}

\author{
R. Feng, ${ }^{1, a)}$ F. Kremer, ${ }^{1}$ D. J. Sprouster, ${ }^{2}$ S. Mirzaei, ${ }^{1}$ S. Decoster, ${ }^{3}$ C. J. Glover, ${ }^{4}$ \\ S. A. Medling, ${ }^{1}$ S. P. Russo, ${ }^{5}$ and M. C. Ridgway ${ }^{1}$ \\ ${ }^{1}$ Department of Electronic Materials Engineering, Research School of Physics and Engineering, \\ Australian National University, Canberra, Australian Capital Territory 0200, Australia \\ ${ }^{2}$ Nuclear Science and Technology Department, Brookhaven National Laboratory, Upton, \\ New York 11973, USA \\ ${ }^{3}$ Instituut voor Kern-en Stralingsfysica, KU Leuven, 3001 Leuven, Belgium \\ ${ }^{4}$ Australian Synchrotron, 800 Blackburn Road, Clayton, Victoria 3168, Australia \\ ${ }^{5}$ Department of Applied Physics, School of Applied Sciences, RMIT University, Melbourne 3001, Australia
}

(Received 10 August 2015; accepted 7 October 2015; published online 22 October 2015)

We report on the effects of dopant concentration on the structural and electrical properties of In-implanted Ge. For In concentrations of $\leq 0.2$ at. $\%$, extended $x$-ray absorption fine structure and $\mathrm{x}$-ray absorption near-edge structure measurements demonstrate that all In atoms occupy a substitutional lattice site while metallic In precipitates are apparent in transmission electron micrographs for In concentrations $\geq 0.6$ at. \%. Evidence of the formation of In-vacancy complexes deduced from extended x-ray absorption fine structure measurements is complimented by density functional theory simulations. Hall effect measurements of the conductivity, carrier density, and carrier mobility are then correlated with the substitutional In fraction. (C) 2015 AIP Publishing LLC.

[http://dx.doi.org/10.1063/1.4934200]

\section{INTRODUCTION}

Ge has increasingly important applications in the fabrication of semiconductor devices due to a high hole mobility ${ }^{1}$ and low dopant activation temperature in comparison with $\mathrm{Si}^{2}$ For this reason, Ge is likely to replace $\mathrm{Si}$ in future high-mobility complementary metal oxide semiconductor (CMOS) devices, ${ }^{3,4}$ where the channel of the $n$-type field-effect transistor (NFET) is based on InGaAs while that of the $p$-type fieldeffect transistor (PFET) is formed from Ge. The $p$-type dopants in Ge have element-specific advantages. The low intrinsic diffusion of $\mathrm{B}$ in $\mathrm{Ge}$ is ideal for ultra-shallow junction formation, ${ }^{5,6}$ while Ga exhibits a very high solid solubility limit in Ge. ${ }^{7}$ Recently, In has been reconsidered as a $p$-type dopant in Ge given it introduces a shallow accepter level $0.0112 \mathrm{eV}$ above the Ge valence band. ${ }^{8}$ Given that the presence of dopants and disorder in a semiconductor lattice influence the electrical properties of a device, ${ }^{1}$ a comprehensive understanding of such influences calls for a detailed study, as presented herein, of In-doped Ge at both the micro and macro scales.

Dopant diffusion and electrical defects in In-doped Ge have previously been investigated as a function of annealing conditions using secondary ion mass spectrometry (SIMS) ${ }^{9}$ and deep level transient spectroscopy (DLTS), ${ }^{10}$ respectively. The latter demonstrated that $\mathrm{Ge}$ point defects are not In related and recover after annealing at $600^{\circ} \mathrm{C}$. Previous studies have shown that most dopant species in Ge tend to occupy substitutional lattice sites and most defect processes are mediated by vacancies (V) (since interstitials (I) have significantly higher formation energy). ${ }^{9,11,12}$ Density Functional Theory (DFT) calculations were performed to predict the binding energy and migration enthalpies of different types of In-vacancy $\left(\operatorname{In}_{\mathrm{m}} \mathrm{V}_{\mathrm{n}}\right)$ clusters. ${ }^{13,14}$ Recent lattice-site

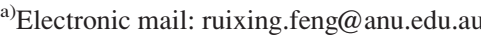

location studies using the emission channeling technique ${ }^{15}$ concluded that In preferentially occupies Ge bond-centered sites before annealing for low In implantation fluences $\left(2.9 \times 10^{12}\right.$ ions $\left./ \mathrm{cm}^{2}\right)$ as consistent with earlier perturbed angular correlation spectroscopy (PAC) measurements ${ }^{16-18}$ and DFT calculations. ${ }^{19}$ After annealing at $300^{\circ} \mathrm{C}$, In atoms then redistribute to substitutional sites. For In concentrations in the range used for impurity doping, complementary information about the local atomic environment around an In atom, including coordination numbers $(\mathrm{CNs})$, structural disorder and bond lengths, is lacking. Such knowledge could enable one to correlate subtle changes in atomic environment to the electrical properties as a function of In concentration.

In this report, we have used the synchrotron-based analytical techniques of $\mathrm{x}$-ray absorption near edge structure (XANES) and extended $\mathrm{x}$-ray absorption fine structure (EXAFS) to study the atomic-scale environment of In-doped Ge over a broad In concentration range. Previously, XANES and EXAFS were utilized to investigate In complexes in $\mathrm{Si}$, demonstrating In atoms were substitutional at low concentrations and precipitated at high concentrations. ${ }^{20,21}$ The current literature is lacking information about In-doped Ge examined with XANES and EXAFS, which we address in this report. Complementary measurements of the structural properties using transmission electron microscopy (TEM) and Raman spectroscopy were also performed as were DFT calculations. Finally, the electrical properties were characterized with Hall effect measurements to enable the correlation of structural and electrical properties of In-doped $\mathrm{Ge}$ at concentrations appropriate for semiconductor doping technologies.

\section{EXPERIMENTAL METHODS}

Nominally undoped Ge layers of thickness $1.8 \mu \mathrm{m}$ were deposited on (100) Si substrates by ultra-high vacuum 
chemical vapour deposition and then implanted with In ions. We used a Ge/Si heterostructure to enable the subsequent removal of the $\mathrm{Si}$ substrate for superior synchrotron-based measurements, as described below. The lattice mismatch between $\mathrm{Ge}$ and $\mathrm{Si}$ yields misfit dislocations in proximity to the $\mathrm{Ge} / \mathrm{Si}$ interface at $1.8 \mu \mathrm{m}$, the influence of which was minimized by confining the In depth distributions to depths $<1.2 \mu \mathrm{m}$. Implantation was performed at $250^{\circ} \mathrm{C}$ to avoid amorphization with the surface normal offset $7^{\circ}$ from the incident ion direction to avoid channeling. Implantation energies ranged from 700 to $3400 \mathrm{keV}$ with fluences varying from $1.79 \times 10^{14}$ to $5.44 \times 10^{16}$ ions $/ \mathrm{cm}^{2}$, as listed in Table I. The given implantation energy/fluence combinations yielded a uniform In depth distribution over $0.2-1.2 \mu \mathrm{m}$ with In concentrations ranging from 0.02 to 1.2 at. \% as calculated with TRIM-2008 (Ref. 22) and confirmed with Rutherford backscattering spectrometry after annealing. A three step annealing process in $\mathrm{N}_{2}$, at temperatures of 550,450 , and $350^{\circ} \mathrm{C}$ for $0.5,1$, and $2 \mathrm{~h}$, respectively, was used to initially activate the implanted In atoms and reduce lattice disorder $\left(550^{\circ} \mathrm{C}\right)$ and then enhance the In-defect concentrations by lowering the solid solubility limit $\left(350^{\circ} \mathrm{C}\right)$.

For the synchrotron-based measurements, we used a unique lift-off technique ${ }^{23}$ to isolate the In-rich Ge layers. The $\mathrm{Si}$ substrate was removed through mechanical grinding and selective wet chemical etching in $\mathrm{KOH}$, then multiple $\mathrm{Ge}$ layers were stacked between two x-ray transparent Kapton windows. As a consequence, a significant amount of material was concentrated within the sample holder, scattering from the $\mathrm{Si}$ substrate was eliminated and high-resolution measurements were enabled. Fluorescence mode XANES and EXAFS measurements were performed at the X-ray absorption spectroscopy beamline of the Australian Synchrotron. In K-edge spectra were recorded with a $10 \times 10$ pixel-array Ge detector with the samples maintained at a temperature of $18 \mathrm{~K}$ to minimize thermal disorder. Multiple scans (3-6) were collected for each sample and averaged to maximize the signal-to-noise ratio. Data were recorded to a photoelectron wavenumber $(k)$ value of $12 \AA^{-1}$. For energy calibration, an In reference foil was simultaneously measured in transmission mode.

Cross-sectional TEM measurements were performed with a Phillips CM300 operating at $300 \mathrm{kV}$. Samples were prepared with conventional methods: mechanical grinding to $80 \mu \mathrm{m}$, dimple grinding to $10-20 \mu \mathrm{m}$ then final polishing using Ar ion milling with the sample maintained at liquid $\mathrm{N}_{2}$ temperature.

Raman spectroscopy measurements were performed using a Jobin-Yvon Horiba T64000 Raman spectrometer, operating in the single spectrometer mode using a liquid $\mathrm{N}_{2}$ cooled CCD detector in the backscattering configuration. Spectra were acquired at room temperature using a short working distance (Nikon, 100×) with a $\mathrm{HeNe}$ laser $(632.8 \mathrm{~nm}$ ) focused to a $2 \mu \mathrm{m}$ diameter spot. Ten spectra per sample were each collected for $30 \mathrm{~s}$ and then averaged. Two different regions of each sample were probed.

Electrical characterization was performed using a LakeShore 7700A Series Hall effect electron transport measurement system at room temperature. To achieve an Ohmic contact, four $0.75 \mathrm{~mm}$ diameter, $200 \mathrm{~nm}$ thick $\mathrm{Al}$ contacts were thermally evaporated on the corners of the sample surface with photoresist as the mask. The Ohmic nature of the contacts was confirmed with IV measurements. The samples were patterned with two different Van Der Pauw geometries: (i) square $\left(1 \times 1 \mathrm{~cm}^{2}\right)$ and (ii) clover leaf (radius $\left.1 \mathrm{~cm}\right){ }^{24,25}$ The clover leaf samples were fabricated by spin coating AZ5214 photoresist and UV light exposure. The samples were then etched in $\mathrm{H}_{2} \mathrm{O}_{2}$ for $4 \mathrm{~h}$ at room temperature to remove the exposed $\mathrm{Ge}$.

Background subtraction, spectra alignment, and normalization of the EXAFS data were performed with ATHENA. ${ }^{26}$ Isolated EXAFS spectra were then Fouriertransformed (FT) over a $k$ range of $2.2-11 \AA^{-1}$ with an adaptive Hanning window and back FT over a non-phasecorrected radial distance $R$ range of $1.6-4.65 \AA(2.3-4 \AA$ for the In standard). Structural parameters were determined with ARTEMIS ${ }^{26}$ utilizing the IFFEFIT package ${ }^{27}$ with theoretical scattering amplitudes and phase shifts calculated $a b$ initio with FEFF8.4. ${ }^{28}$ The experimental data were fitted using $k$-weights of 2,3 , and 4 simultaneously, with a complete multiple scattering analysis. The parameters used in the triple $k$-weights fit were listed in Table II, while the spectra were plotted with $k$-weight of 2 in Figures 2 and 3. Four different models were used to characterize the atomicscale environment of implanted In atoms as shown schematically in Figure 1:

(1) All In atoms occupying a substitutional lattice position in Ge;

(2) All In atoms are in a metallic In environment;

(3) A combination of (1) and (2);

(4) As per (3) but with the addition of a vacancy in the first shell surrounding an In atom in Ge.

The amplitude reduction factor $S_{0}^{2}$ was determined for the In metal standard (0.7) and then fixed for the subsequent fitting of all spectra. The energy shift parameter $\left(\mathrm{E}_{0}\right)$ was fixed to be $1.52 \mathrm{eV}$ for model (1) and $2.15 \mathrm{eV}$ for model (2),

TABLE I. Implantation fluences (atoms $/ \mathrm{cm}^{2}$ ) and energies for each sample in different In concentrations.

\begin{tabular}{|c|c|c|c|c|c|}
\hline $\begin{array}{r}\text { Conce } \\
\text { (at. \% }\end{array}$ & 0.02 & 0.06 & 0.2 & 0.6 & 1.2 \\
\hline 700 & $1.19 \times 10^{14}$ & $3.58 \times 10^{14}$ & $1.19 \times 10^{15}$ & $3.58 \times 10^{15}$ & $7.15 \times 10^{15}$ \\
\hline 1400 & $2.06 \times 10^{14}$ & $6.19 \times 10^{14}$ & $2.06 \times 10^{15}$ & $6.19 \times 10^{15}$ & $1.24 \times 10^{16}$ \\
\hline 2300 & $2.34 \times 10^{14}$ & $7.02 \times 10^{14}$ & $2.34 \times 10^{15}$ & $7.02 \times 10^{15}$ & $1.40 \times 10^{16}$ \\
\hline 3400 & $5.44 \times 10^{14}$ & $1.63 \times 10^{15}$ & $5.44 \times 10^{15}$ & $1.63 \times 10^{16}$ & $3.26 \times 10^{16}$ \\
\hline
\end{tabular}


TABLE II. EXAFS fitting results as a function of In concentration and model. $\alpha$ is the factor of difference between the calculated and theoretical In path lengths; $\sigma_{I n}^{2}$ is the DWF used in the fittings with model (2); $\mathrm{r}_{1}, \mathrm{r}_{2}, \mathrm{r}_{3}$ and $\sigma_{1}^{2}, \sigma_{2}^{2}, \sigma_{3}^{2}$ are the radial distances to the first, second, and third NNs and their corresponding DWFs, respectively; $\mathrm{f}_{\mathrm{m}}(\%)$ is the In metal fraction and $1 \mathrm{st} \mathrm{NN} \mathrm{CN}_{\mathrm{Ge}}$ is the coordination number of Ge surrounding In in the first shell; R-factor represents the fit quality; uncertainties are given in brackets.

\begin{tabular}{|c|c|c|c|c|c|c|c|c|c|c|c|c|}
\hline Sample & Model & $\alpha(\%)$ & $\sigma_{I n}^{2}\left(\AA^{2}\right)$ & $\mathrm{r}_{1}(\AA)$ & $\mathrm{r}_{2}(\AA)$ & $r_{3}(\AA)$ & $\sigma_{1}^{2}\left(\AA^{2}\right)$ & $\sigma_{2}^{2}\left(\AA^{2}\right)$ & $\sigma_{3}^{2}\left(\AA^{2}\right)$ & $\mathrm{f}_{\mathrm{m}}(\%)$ & 1 st $\mathrm{NN} \mathrm{CN}_{\mathrm{Ge}}$ & R-factor \\
\hline $\begin{array}{l}0.02 \\
\text { at. \% }\end{array}$ & (1) & $\ldots$ & $\ldots$ & $\begin{array}{c}2.558 \\
(2)\end{array}$ & $\begin{array}{c}4.042 \\
(4)\end{array}$ & $\begin{array}{c}4.683 \\
(8)\end{array}$ & $\begin{array}{c}0.0032 \\
\text { (3) }\end{array}$ & $\begin{array}{c}0.0041 \\
\text { (4) }\end{array}$ & $\begin{array}{c}0.0064 \\
(9)\end{array}$ & $\ldots$ & 4 & 0.0093 \\
\hline $\begin{array}{l}0.06 \\
\text { at. } \%\end{array}$ & (1) & $\ldots$ & $\ldots$ & $\begin{array}{c}2.559 \\
(2)\end{array}$ & $\begin{array}{c}4.046 \\
\text { (3) }\end{array}$ & $\begin{array}{c}4.685 \\
(7)\end{array}$ & $\begin{array}{c}0.0034 \\
\text { (2) }\end{array}$ & $\begin{array}{c}0.0046 \\
\text { (4) }\end{array}$ & $\begin{array}{c}0.0071 \\
(9)\end{array}$ & $\ldots$ & 4 & 0.0078 \\
\hline $\begin{array}{l}0.2 \\
\text { at. } \%\end{array}$ & (1) & $\ldots$ & $\ldots$ & $\begin{array}{c}2.563 \\
(1)\end{array}$ & $\begin{array}{c}4.053 \\
\text { (3) }\end{array}$ & $\begin{array}{c}4.689 \\
(7)\end{array}$ & $\begin{array}{c}0.0036 \\
\text { (2) }\end{array}$ & $\begin{array}{c}0.0051 \\
\text { (3) }\end{array}$ & $\begin{array}{c}0.0074 \\
(7)\end{array}$ & $\ldots$ & 4 & 0.0055 \\
\hline $\begin{array}{l}0.2 \\
\text { at. \% }\end{array}$ & (3) & $\begin{array}{c}-7.36 \\
(24)\end{array}$ & $\begin{array}{c}0.0293 \\
(17)\end{array}$ & $\begin{array}{c}2.562 \\
(1)\end{array}$ & $\begin{array}{c}4.051 \\
\text { (3) }\end{array}$ & $\begin{array}{c}4.690 \\
(5)\end{array}$ & $\begin{array}{c}0.0026 \\
(1)\end{array}$ & $\begin{array}{c}0.0043 \\
\text { (3) }\end{array}$ & $\begin{array}{c}0.0067 \\
(7)\end{array}$ & $\begin{array}{l}14 \\
(7)\end{array}$ & 4 & 0.0038 \\
\hline $\begin{array}{l}0.6 \\
\text { at. } \%\end{array}$ & (3) & $\begin{array}{c}-2.15 \\
(12)\end{array}$ & $\begin{array}{c}0.0052 \\
(5)\end{array}$ & $\begin{array}{c}2.561 \\
(7)\end{array}$ & $\begin{array}{c}4.037 \\
(9)\end{array}$ & $\begin{array}{c}4.711 \\
(29)\end{array}$ & $\begin{array}{c}0.0042 \\
(8)\end{array}$ & $\begin{array}{c}0.0031 \\
(10)\end{array}$ & $\begin{array}{c}0.0060 \\
(19)\end{array}$ & $\begin{array}{l}72 \\
\text { (7) }\end{array}$ & 4 & 0.0188 \\
\hline $\begin{array}{l}0.6 \\
\text { at. } \%\end{array}$ & (4) & $\begin{array}{c}-2.12 \\
(9)\end{array}$ & $\begin{array}{c}0.0041 \\
(4)\end{array}$ & $\begin{array}{c}2.561 \\
(5)\end{array}$ & $\begin{array}{c}4.044 \\
(8)\end{array}$ & $\begin{array}{c}4.712 \\
(14)\end{array}$ & $\begin{array}{c}0.0036 \\
(5)\end{array}$ & $\begin{array}{c}0.0055 \\
(8)\end{array}$ & $\begin{array}{c}0.0071 \\
(13)\end{array}$ & $\begin{array}{l}63 \\
(4)\end{array}$ & $\begin{array}{c}3.1 \\
(0.4)\end{array}$ & 0.0118 \\
\hline $\begin{array}{l}1.2 \\
\text { at. } \%\end{array}$ & (3) & $\begin{array}{c}-2.13 \\
(11)\end{array}$ & $\begin{array}{c}0.0050 \\
(5)\end{array}$ & $\begin{array}{c}2.561 \\
(14)\end{array}$ & $\begin{array}{c}4.034 \\
(17)\end{array}$ & $\begin{array}{c}4.739 \\
(36)\end{array}$ & $\begin{array}{c}0.0044 \\
(12)\end{array}$ & $\begin{array}{c}0.0027 \\
(15)\end{array}$ & $\begin{array}{c}0.0047 \\
(25)\end{array}$ & $\begin{array}{l}81 \\
(6)\end{array}$ & 4 & 0.0174 \\
\hline $\begin{array}{l}1.2 \\
\text { at. } \%\end{array}$ & (4) & $\begin{array}{c}-2.13 \\
(9)\end{array}$ & $\begin{array}{c}0.0041 \\
\text { (3) }\end{array}$ & $\begin{array}{c}2.561 \\
(10)\end{array}$ & $\begin{array}{c}4.033 \\
(12)\end{array}$ & $\begin{array}{c}4.732 \\
(21)\end{array}$ & $\begin{array}{c}0.0039 \\
(7)\end{array}$ & $\begin{array}{c}0.0056 \\
(11)\end{array}$ & $\begin{array}{c}0.0068 \\
(15)\end{array}$ & $\begin{array}{l}72 \\
(5)\end{array}$ & $\begin{array}{c}2.6 \\
(0.5)\end{array}$ & 0.0130 \\
\hline In foil & (2) & $\begin{array}{c}-0.46 \\
(6)\end{array}$ & $\begin{array}{c}0.0040 \\
\text { (2) }\end{array}$ & $\ldots$ & $\ldots$ & $\ldots$ & $\ldots$ & $\ldots$ & $\ldots$ & 100 & $\ldots$ & 0.0046 \\
\hline
\end{tabular}

according to the fitting quality. For model (1), three different Debye-Waller Factors (DWFs) and bondlengths were used for the single scattering paths. DWFs and bondlengths of the multiple scattering paths were calculated from single scattering paths to reduce the number of variables. ${ }^{29,30}$ Since the two main scattering paths of In metal are similar (3.245 and $3.370 \AA$ ), analysis using model (2) floated all the bondlengths by multiplying the theoretical values by a factor $(1+\alpha)$, $\mathrm{R}=\operatorname{Reff} \times(1+\alpha)$, and a single DWF variable was used. Model (3) was achieved by combining the scattering amplitude of model (2) multiplying by a metal fraction $\mathrm{f}_{\mathrm{m}}$ and model (1) multiplying by a substitutional fraction, $1-\mathrm{f}_{\mathrm{m}}$. Model (4) is the same as model (3) but with a reduced CN of the first shell surrounding an In atom in Ge.

To aid in fitting the EXAFS results and simulating the XANES spectra, DFT calculations for a substitutional In atom with and without a vacancy in the first shell were performed using VASP. ${ }^{31}$ The generalized-gradient approximation (GGA) with the Perdew-Burke-Ernzerhof (PBE) exchange-correlation function ${ }^{32}$ was utilized to calculate the interaction between the In atom, vacancy, and the $2 \times 2 \times 2$ Ge supercell, with a $10 \times 10 \times 10$ k-point mesh using the
Monkhorst-Pack sampling scheme. ${ }^{33}$ The projector augmented wave (PAW) pseudopotentials ${ }^{34}$ implemented in VASP were also used with a planewave cutoff energy of $240 \mathrm{eV}$. A conjugate-gradient (CG) geometry optimization algorithm was used to relax the lattice following the progressive relaxation procedure: ${ }^{35}$ Ion relaxation was first performed between the dopant(s) and the surrounding atoms over an increasing region size followed by a cell parameter relaxation and then the final relaxation of all atoms, cell volume, and cell shape. Ab-initio molecule dynamics (MD) runs in VASP were also performed from 1000 to $300 \mathrm{~K}$ with 1000 ionic steps under the RMM-DIIS scheme ${ }^{36}$ before the DFT geometry optimization to simulate an annealing process and assure a global minimum total energy was obtained in the subsequent DFT calculation.

$A b$-initio XANES calculations of substitutional In (with structural relaxation performed in DFT) and In metal were performed for comparison with the experimental results. FEFF9 $^{37}$ was used for a full multiple scattering analysis for all paths within a radius of $8.5 \AA$ (123 atoms) for substitutional In and $9.5 \AA$ (139 atoms) for metallic In. Calculations with larger cluster radii confirmed the spectra fully
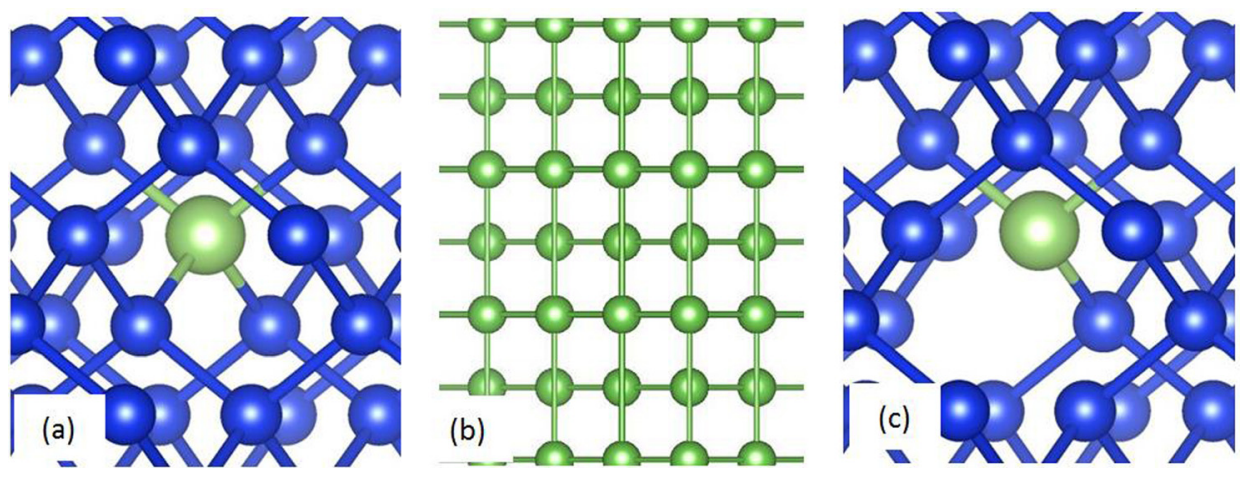

FIG. 1. Structural models used in the EXAFS fitting showing (a) a substitutional In atom in a Ge lattice, (b) metallic In, (c) a substitutional In atom in a Ge lattice with a vacancy in the first NN shell. 


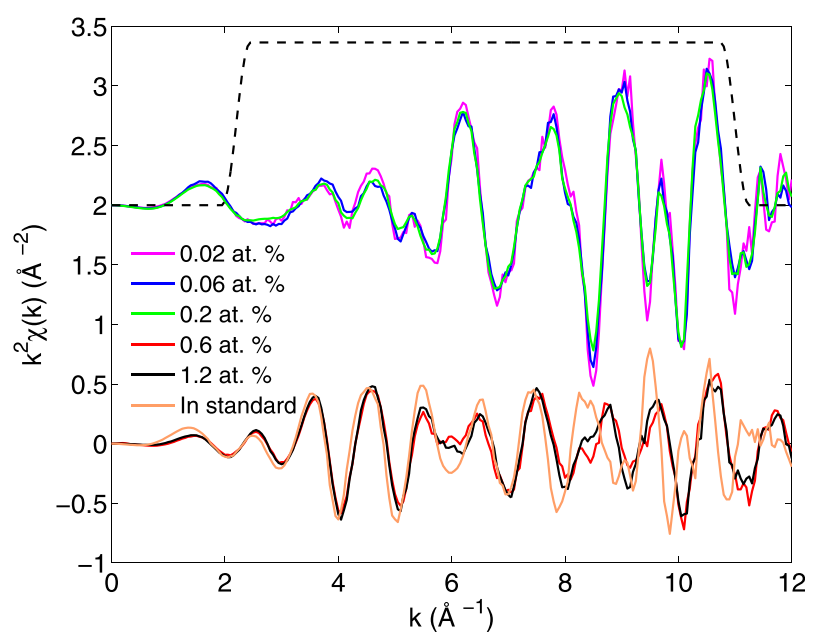

FIG. 2. $\mathrm{k}^{2}$-weighted EXAFS spectra as a function of photoelectron wavenumber for In concentrations of $0.02-1.2$ at. \%. Spectra are offset vertically for clarity. Dashed line shows the k-range window used for the data fitting.

converged at the given radii. These calculations were based on overlapping muffin-tin potentials obtained from selfconsistent calculations with a radius equal to $6 \AA$ (35 atoms for substitutional In and 43 atoms for metallic In) using the Hedin Lundqvist exchange function. ${ }^{38}$ The amplitude reduction factor $\left(S_{0}^{2}\right)$ used in these calculations was 0.7 based on the EXAFS fittings. Complementary FDMNES calculations ${ }^{39}$ were also performed using a similar multiple scattering formalism within the muffin-tin approximation (MTA). The MTA was chosen over the finite difference method (FDM) given it was computationally much less demanding. The FDM results were very similar to those from MTA since they use the same package but with different potentials. Full multiple scattering calculations were performed within the same radius used for the FEFF9 simulations and convergence was also confirmed.

\section{RESULTS}

Figure 2 shows EXAFS spectra as a function of In concentration. The $0.02,0.06$, and 0.2 at. \% samples share a similar EXAFS spectrum, while the 0.6 and 1.2 at. \% samples are comparable to the In standard. This implies that In atoms have a common atomic environment for In concentrations $\leq 0.2$ at. $\%$ and begin to precipitate to form metallic In for concentrations of $>0.6$ at. $\%$. Fittings of the FT EXAFS

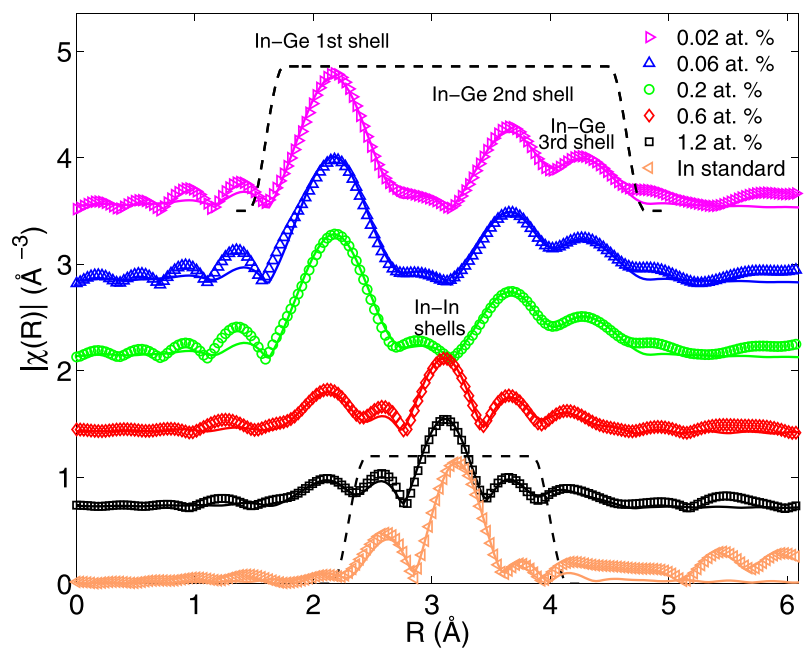

FIG. 3. FT EXAFS spectra as a function of radial distance for In concentrations of 0.02-1.2 at. \%. Spectra are offset vertically for clarity. Dashed lines show the R-range windows used for the data fitting. Symbols represent the data while solid lines are best fits using model (1) for $0.02,0.06$, and 0.2 at. \%, model (2) for the In standard, and model (4) for 0.6 and 1.2 at. \%.

spectra, as shown in Figure 3, confirm this argument. For samples with In concentration $\leq 0.2$ at. $\%$, In atoms occupy a four-fold coordinated substitutional site in a crystalline $\mathrm{Ge}$ matrix, consistent with model (1). For these three samples, the In-Ge nearest neighbor (NN) distances (Figure 4(a)) increase with increasing In concentration. The surrounding Ge lattice expands by $\sim 0.1$ and $0.05 \AA$ at the first and second shells, respectively, due to the presence of substitutional In. The fitted DWFs (Figure 4(b)) for these three samples also increase as a function of In concentration, indicative of increasing disorder. Fitting the 0.2 at. \% sample spectrum with model (3) yielded no evidence of a two component system comprising substitutional In and metallic In.

For 0.6 and 1.2 at. \% samples, the FT EXAFS spectra appear to be comprising substitutional In and metallic In components. However, application of model (3) to the EXAFS analysis, where a substitutional In atom is fourfold coordinated with Ge atoms, yielded non-physical DWFs (relatively high for the first shell but unrealistically low for the second and third shells) (Figure 4(b)). Given the DWF and CN are highly correlated and both influence the FT EXAFS magnitude, model (4) was invoked where the $\mathrm{CN}$ for In atoms in the non-metallic environment was floated such that $\mathrm{CN} \leq 4$ atoms. The resulting $\mathrm{R}$ values (the

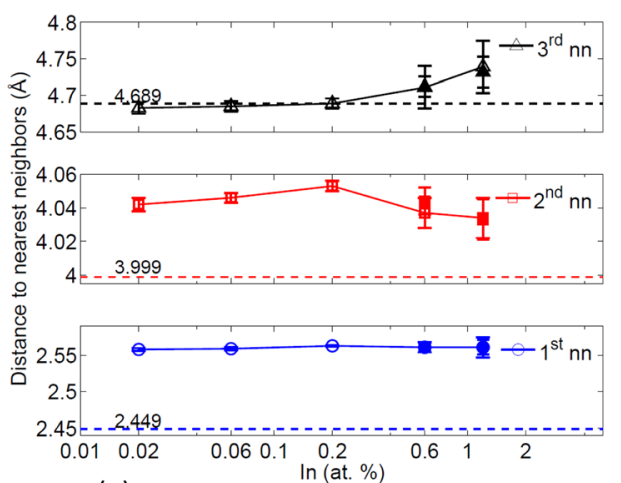

(a)

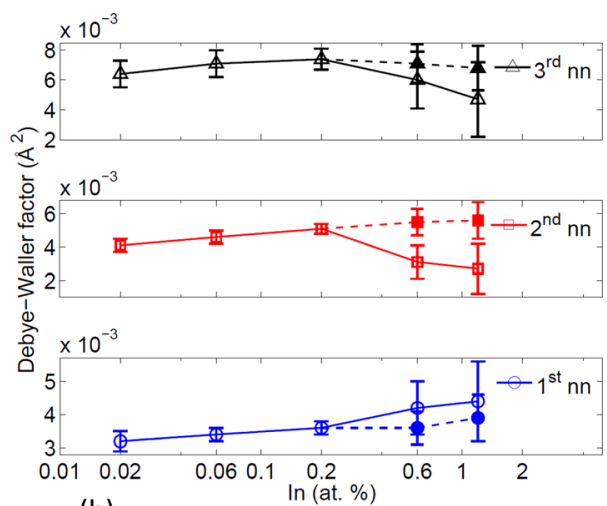

(b)
FIG. 4. (a) Radial distances and (b) DWFs from the In atom to the first (circles), second (squares), and third (triangles) $\mathrm{NN}$ determined with EXAFS. For $0.02,0.06$, and 0.2 at. $\%$ samples, fitting results with model 1 are shown for 0.6 and 1.2 at. \% samples, fitting results with models 3 (open symbols) and 4 (solid symbols) are shown. Dashed lines on (a) are the $\mathrm{NN}$ radial distances in bulk Ge. 

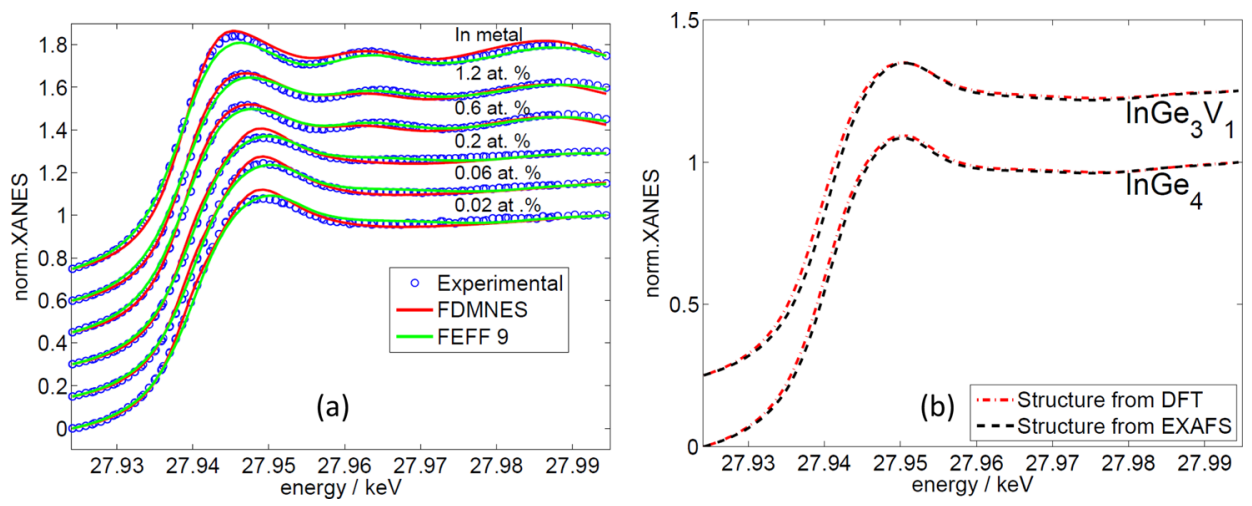

FIG. 5. (a) XANES spectra (circles) and their best fits with FDMNES (dashed lines) and FEFF9 (dot dashed lines) using the LCF method. Metal fractions are listed in Table III. The fittings basing on In metal combining In substitution Ge with 1st NNs coordination numbers 3 were plotted for 0.6 at. \% and 1.2 at. \% samples. (b) The comparison of simulated XANES spectra with coordination numbers 3 and 4 in the In to Ge first NNs and the simulated XANES spectra from EXAFS modeling and DFT structure relaxation. All spectra were vertically offset for clarity.

relative error of the fit to the data) were much improved, attesting to the higher validity of this model compared to that of model (3).

The fittings of 0.6 and 1.2 at. \% samples with model (4) yielded metallic fractions of 63 and $72 \%$, respectively, with substitutional In atom $\mathrm{CNs}$ of 3.1 and 2.6 atoms, respectively. The dashed lines of Figure 4(b) demonstrate that the fitted DWFs for the non-metallic In component of these two samples with model (4) are, within experimental error, effectively constant as a function In concentration, as is the In-Ge $\mathrm{NN}$ distance, while the distances to second and third NNs (Figure 4(a)) decrease and increase, respectively, at 0.6 at. \% and beyond. We speculate the reduced $\mathrm{CN}$ and reduced second NN distance are evidence of an In-V complex. Comparing the fitting of the metallic component to the In standard, the In-In NN distance in the former is reduced by $2 \%$. This implies that the In precipitates in the 0.6 and 1.2 at. \% samples are compressed by the Ge matrix. The $\mathrm{CN}$ for the In precipitates and In standard was equal, indicating that finite-sizes effects were negligible and thus the In precipitates must be of a significant size $(\gtrsim 10 \mathrm{~nm})$. The EXAFS spectra fitting results are listed in Table II. We acknowledge that the fitting results from model (4), an In atom in a Ge lattice with one NN removed, could potentially be improved by instead using the DFT relaxed structure. We chose however not to use the DFT relaxed structure for simplicity and to retain continuity between the models.

The x-ray absorption near-edge structure (XANES) spectra were normalized from $15 \mathrm{eV}$ below to $55 \mathrm{eV}$ above the In $\mathrm{K}$-edge and are shown in Figure 5(a). As the In concentration increases, the absorption edges are shifted to lower energy, toward the metallic state, as expected. The XANES spectra of the 0.6 and 1.2 at. \% samples exhibit an oscillation characteristic of In metal but with lower amplitude given the metallic In fraction in these samples is $<100 \%$. The XANES spectra for samples with lower In concentrations (0.02-0.2 at. \%) are by comparison much smoother.

The simulated spectra of substitutional In in a Ge lattice, with and without vacancies, and In metal were normalized and aligned to the experimental spectra. The simulated spectra then served as standards to perform linear combination fitting (LCF) of the experimental results.
A LCF was also performed with the ATHENA program using the experimental XANES spectra of the 0.02 at. \% and In standard. The calculated In metal fractions are listed in Table III. Figure 5(a) shows that the experimental results are well described by the combination of substitutional In in Ge and In metal models, and the two simulation methods agree well. Figure 5(b) shows that the simulated XANES spectra are insensitive to the presence of a vacancy in the first NN shell surrounding a substitutional In atom. The variation from one simulation code to another (FEFF9 and FDMMES) is greater than the variation with and without a vacancy using one of the two given codes. As shown in Table III, the calculated In metal fractions from the Athena LCF based on experimental spectra generally agree with the fitting based on simulated spectra from FDMNES and FEFF9 and also the results from EXAFS fitting.

The In-Ge NN distance calculated by DFT, with and without a vacancy, agrees within $2 \%$ with the EXAFS results. Figure 5(b) shows that the XANES spectra simulated from the DFT relaxed structure are in good agreement with the structure from the models derived with EXAFS. The high quality of the XANES fitting also implies that the models are physically realistic. Other models, such as two vacancies or an In atom in the first NN shell, were also

TABLE III. In metal fractions as a function of In concentration comparing results from experimental XANES (ATHENA), simulated XANES (FDMNES and FEFF9), and experimental EXAFS. The first NN shell surrounding an In atom comprised Ge atoms with the coordination number designated "1st $\mathrm{NN} \mathrm{CN}_{\mathrm{Ge}}$." Uncertainties are given in parentheses.

\begin{tabular}{lccccc}
\hline \hline Sample & 1 st NN CN $\mathrm{Ge}$ & ATHENA & FDMNES & FEFF9 & EXAFS \\
\hline 0.02 at. $\%$ & 4 & 0 & $0(3) \%$ & $0(2) \%$ & $0 \%$ \\
0.06 at. $\%$ & 4 & $0(2) \%$ & $0(1) \%$ & $3(1) \%$ & $0 \%$ \\
0.2 at. $\%$ & 4 & $15(1) \%$ & $11(1) \%$ & $16(1) \%$ & $0 \%$ \\
0.6 at. $\%$ & 4 & $62(2) \%$ & $61(3) \%$ & $61(2) \%$ & $72(7) \%$ \\
0.6 at. $\%$ & 3 & $\ldots$ & $59(2) \%$ & $60(3) \%$ & $63(4) \%$ \\
1.2. at. $\%$ & 4 & $70(1) \%$ & $69(3) \%$ & $70(1) \%$ & $81(6) \%$ \\
1.2 at. $\%$ & 3 & $\ldots$ & $66(2) \%$ & $67(3) \%$ & $72(5) \%$ \\
\hline \hline
\end{tabular}

${ }^{\mathrm{a}}$ The $1 \mathrm{st} \mathrm{NN} \mathrm{CN}_{\mathrm{Ge}}$ value determined with EXAFS is 3.1 for this sample. ${ }^{\mathrm{b}}$ The $1 \mathrm{st} \mathrm{NN} \mathrm{CN}_{\mathrm{Ge}}$ value determined with EXAFS is 2.6 for this sample. 
investigated with DFT but were not compatible with either the EXAFS or XANES results.

Figure 6 shows TEM images as a function of In concentration. No structural disorder or metallic In precipitates are observed at low In concentration (0.06 at. \%), while precipitates are apparent for In concentrations of $>0.6$ at. \%. The precipitate density and size are greater for the 1.2 at. \% sample. The crystalline nature of the precipitates is confirmed by the Moire patterns formed as a result of the overlap of the In and Ge lattices. The TEM images thus confirm the

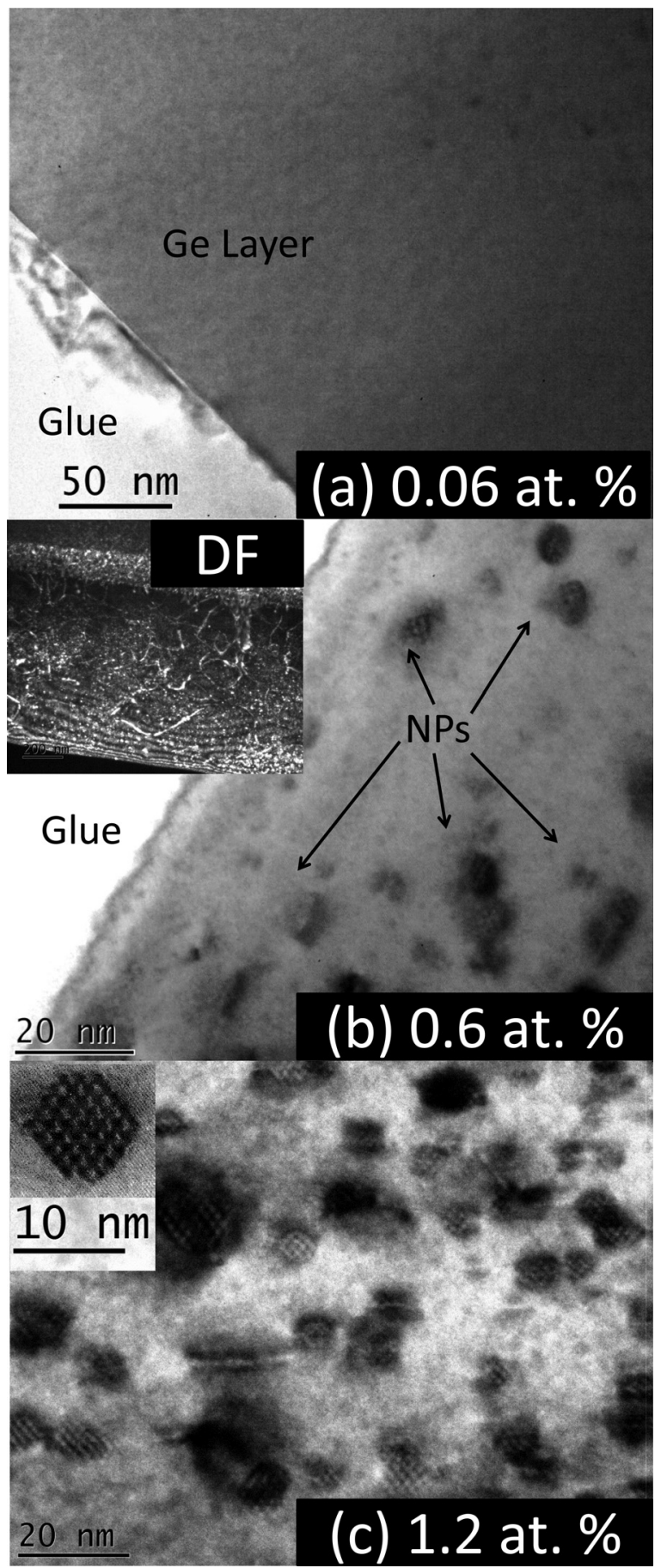

FIG. 6. Cross section TEM images for (a) 0.06 at. \%, (b) 0.6 at. \%, and (c) 1.2 at. \% samples. The inset in (b) is a dark field image while that in (c) shows a high resolution image of a metallic In precipitate. synchrotron-based measurements, showing that In precipitates at $>0.6$ at. \% . At these concentrations, line defects (dislocations) are also visible in the dark-field TEM image (inset in Figure 6(b)).

Raman spectra were also recorded across the In concentration range, focusing on the Ge TO band. (Metallic In is not Raman active.) Figure 7 shows normalized Ge TO spectra fitted with Lorentzian functions. The spectra of the low concentration samples (0.02-0.2 at.\%) are similar to bulk crystalline $\mathrm{Ge}$, while the high concentration samples ( 0.6 and 1.2 at. \%) have much lower amplitude and are shifted to lower wavenumber. Figures 7(b) and 7(c) show the Raman shift and FWHM. A significant change is observed between samples with In concentrations of 0.2 at. $\%$ and 0.6 at. \% (without and with In precipitates). The shift to lower wavenumbers results from the Ge lattice expansion, while the increase in FWHM (and decrease in peak height) results from increasing disorder. The Raman shift and FWHM both scale as a function of In concentration. We also note that no broad features from an amorphous component are observable.

Hall Effect measurements are shown in Figure 8. All samples exhibited $p$-type conductivity and the sample geometry did not significantly influence the results. As the In concentration increases, the resistivity decreases, while the carrier density increases as expected. The carrier mobility decreases as the In concentration increases due to the increase in both disorder and ionized impurity scattering. For high In concentrations ( $>0.6$ at. \%), the In atom active fraction drops significantly. The increase in carrier density continues despite our observation of In precipitates in these samples. We would anticipate a saturation of the carrier density once the In concentration exceeds the solid solubility limit and In precipitates begin to form. This result may indicate that disorder in the Ge lattice can potentially enhance the carrier density, as proposed by Romano et al. ${ }^{40}$

Our results agree well with previous experiments performed by Decoster et al. ${ }^{10,15}$ for low fluence $\left(10^{12} \mathrm{In} / \mathrm{cm}^{2}\right)$ In-doped Ge samples implanted at room temperature. Utilizing DLTS and emission channeling, they showed that defects in the Ge lattice were removed and In atoms occupied a substitutional lattice site after annealing above $500^{\circ} \mathrm{C}$. Rutherford backscattering spectrometry/channeling measurements also demonstrated that most In atoms occupied a substitutional lattice site after annealing ${ }^{41,42}$ in these low fluence samples. Finally, PAC experiments showed In atoms occupied substitutional lattice sites with perfect cubic symmetry after annealing at $600{ }^{\circ} \mathrm{C} .{ }^{16-18}$

Using DFT, we also calculated the binding energy of an In atom and vacancy with the vacancy as either the first, second, or third NN of an In atom. Results are listed in Table IV and agree well with earlier calculations from Chroneos et al..$^{13}$ The negative binding energy is indicative of greater stability when an In atom and vacancy are bound together compared to isolated individually. Our results, and those of Chroneos et al., show that the binding energy increases when the vacancy is either a second or third NN, demonstrating an In- $\mathrm{V}$ cluster is most stable with the vacancy as the first $\mathrm{NN}$. 

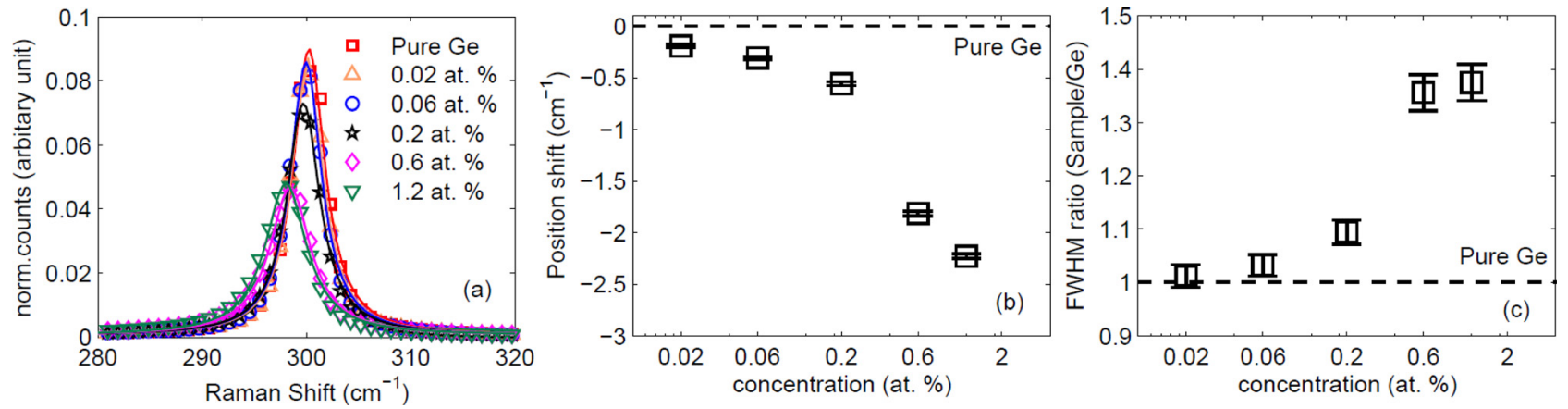

FIG. 7. (a) Raman spectra with data and fits shown as symbols and lines, respectively, (b) peak position shift, and (c) FWHM as a function of In concentration.
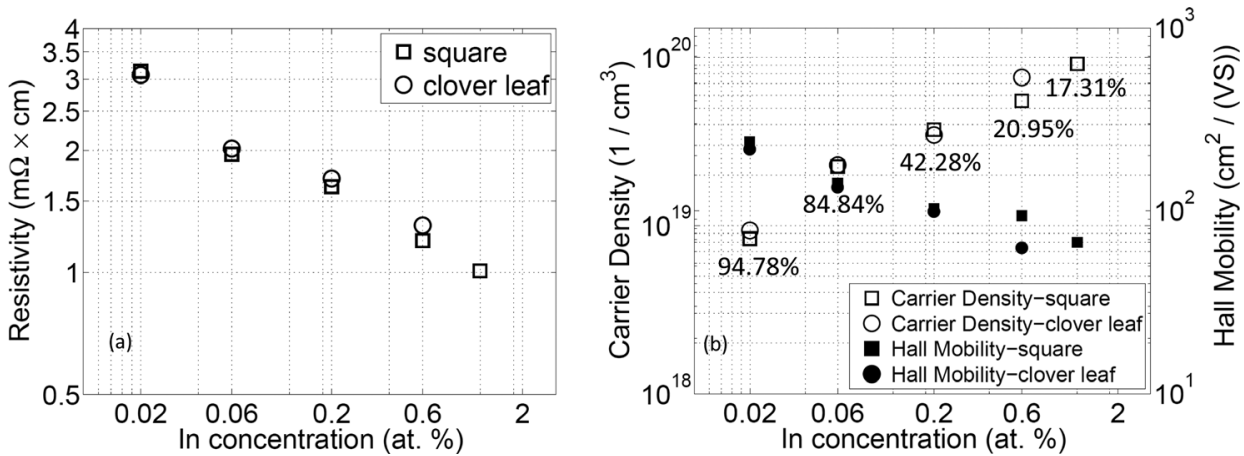

FIG. 8. (a) Resistivity and (b) carrier density and Hall mobility as a function of In concentration, where squares and circle represent square and clover leaf measurement geometries, respectively. The number under the carrier density is the ratio of active to total implanted In atoms (according to measurements on samples of clover leaf patterns).

TABLE IV. Calculated binding energies of an In atom and vacancy with the vacancy as either the first, second, or third NN of an In atom.

\begin{tabular}{lccc}
\hline \hline & 1st NN & 2nd NN & 3rd NN \\
\hline Our calculation & -0.88 & -0.74 & 0.22 \\
Chroneos $^{\text {a }}$ & -0.96 & -0.16 & 0.14 \\
\hline \hline
\end{tabular}

${ }^{\mathrm{a}}$ Reference 13.

This finding clearly supports our EXAFS results where we presented evidence of such an In-V cluster.

\section{CONCLUSIONS}

Our detailed investigation of the atomic-scale environment surrounding an In atom in In-implanted Ge has demonstrated that In atoms occupy a substitutional site at low concentrations and precipitate to form metallic In at high concentrations. In addition, EXAFS measurements yielded evidence of an In-V complex and this result was supported by complementary DFT calculations as it shows In-V pairing is energetically favorable. The synchrotron-based structural characterization was supplemented by TEM and Raman measurements which showed that lattice disorder increases with implanted ion fluence or, equivalently, In concentration. Finally, electrical characterization demonstrated that lattice disorder may enhance the carrier density as suggested in previous reports. ${ }^{40}$

\section{ACKNOWLEDGMENTS}

We acknowledge access to NCRIS and AMMRF infrastructure at the Australian National University, including the Australian National Fabrication Facility, the
Heavy Ion Accelerator Capability, and the Center for Advanced Microscopy. We also thank the Australian Research Council and the Australian Synchrotron for support.

${ }^{1}$ S. M. Sze, Physics of Semiconductor Devices (Wiley, New York, 2001). ${ }^{2}$ R. Hull and J. C. Bean, Germanium Silicon: Physics and Materials, Semiconductors and Semimetals (Academic, San Diego, 1999).

${ }^{3}$ C. C. Yeo, B. J. Cho, F. Gao, S. J. Lee, M. H. Lee, C. Y. Yu, C. W. Liu, L. J. Tang, and T. W. Lee, IEEE Electron Device Lett. 26, 761 (2005).

${ }^{4}$ Y. J. Yang, W. S. Ho, C. F. Huang, S. T. Chang, and C. W. Liu, Appl. Phys. Lett. 91, 102103 (2007).

${ }^{5}$ E. Bruno, G. Impellizzeri, S. Mirabella, A. M. Piro, A. Irrera, and M. G. Grimaldi, Mater. Sci. Eng., B 154-155, 56 (2008).

${ }^{6}$ G. Impellizzeri, S. Mirabella, E. Bruno, A. M. Piro, and M. G. Grimaldi, J. Appl. Phys. 105, 063533 (2009).

${ }^{7}$ E. Simoen and C. Claeys, Germanium-Based Technologies: From Materials to Devices (Elsevier, Amsterdam, 2007).

${ }^{8}$ T. H. Geballe and F. J. Morin, Phys. Rev. 95, 1085 (1954).

${ }^{9}$ R. Kube, H. Bracht, A. Chroneos, M. Posselt, and B. Schmidt, J. Appl. Phys. 106, 063534 (2009).

${ }^{10}$ F. D. Auret, P. J. Janse van Rensburg, M. Hayes, J. M. Nel, W. E. Meyer, S. Decoster, V. Matias, and A. Vantomme, Appl. Phys. Lett. 89, 152123 (2006).

${ }^{11}$ D. J. Sprouster, C. Campbell, S. J. Buckman, G. Impellizzeri, E. Napolitani, S. Ruffell, and J. P. Sullivan, J. Phys. D: Appl. Phys. 46, 505310 (2013).

${ }^{12}$ M. Naganawa, Y. Shimizu, M. Uematsu, K. M. Itoh, K. Sawano, Y. Shiraki, and E. E. Haller, Appl. Phys. Lett. 93, 191905 (2008).

${ }^{13}$ A. Chroneos, H. Bracht, R. W. Grimes, and B. P. Uberuaga, Appl. Phys. Lett. 92, 172103 (2008).

${ }^{14}$ A. Chroneos, R. Kube, H. Bracht, R. W. Grimes, and U. Schwingenschlögl, Chem. Phys. Lett. 490, 38 (2010).

${ }^{15}$ S. Decoster, B. De Vries, U. Wahl, J. G. Correia, and A. Vantomme, J. Appl. Phys. 105, 083522 (2009).

${ }^{16}$ U. Feuser, R. Vianden, and A. F. Pasquevich, Hyperfine Interact. 60, 829 (1990).

${ }^{17}$ H. Haesslein, R. Sielemann, and C. Zistl, Phys. Rev. Lett. 80, 2626 (1998).

${ }^{18}$ R. Sielemann, H. Hässlein, C. Zistl, M. Müller, L. Stadler, and V. V. Emtsev, Physica B 380-310, 529 (2001). 
${ }^{19}$ H. Höhler, N. Atodiresei, K. Schroeder, R. Zeller, and P. H. Dederichs, Phys. Rev. B 71, 035212 (2005).

${ }^{20}$ F. d' Acapito, Y. Shimizu, S. Scalese, M. Italia, P. Alippi, and S. Grasso, Appl. Phys. Lett. 88, 212102 (2006).

${ }^{21}$ F. d' Acapito, Y. Shimizu, S. Scalese, M. Italia, P. Alippi, and S. Grasso, Nucl. Instrum. Methods Phys. Res., Sect. B 253, 59 (2006).

${ }^{22}$ J. F. Ziegler and J. M. Manoyan, Nucl. Instrum. Methods Phys. Res., Sect. B 35, 215 (1988).

${ }^{23}$ S. Decoster, C. J. Glover, B. Johannessen, R. Giulian, D. J. Sprouster, P. Kluth, L. L. Araujo, Z. S. Hussain, C. Schnohr, H. Salama, F. Kremer, K. Temst, A. Vantomme, and M. C. Ridgway, J. Synchrotron Radiat. 20, 426 (2013).

${ }^{24}$ L. J. van der Pauw, Philips Res. Rep. 13, 1 (1958).

${ }^{25}$ H. J. van Daal, Philips Res. Rep., Suppl. 3, 23 (1965).

${ }^{26}$ B. Ravel and M. Newville, J. Synchrotron Radiat. 12, 537 (2005).

${ }^{27}$ M. Newville, J. Synchrotron Radiat. 8, 322 (2001).

${ }^{28}$ A. L. Ankudinov, B. Ravel, J. J. Rehr, and S. D. Conradson, Phys. Rev. B 58, 7565 (1998).

${ }^{29}$ S. Decoster, B. Johannessen, C. J. Glover, S. Cottenier, T. Bierschenk, H. Salama, F. Kremer, K. Temst, A. Vantomme, and M. C. Ridgway, Appl. Phys. Lett. 101, 261904 (2012).
${ }^{30}$ P. Shanthakumar, M. Balasubramanian, D. M. Pease, A. I. Frenkel, D. M. Potrepka, V. Kraizman, J. I. Budnick, and W. A. Hines, Phys. Rev. B 74, 174103 (2006).

${ }^{31}$ G. Kresse and J. Furthmüller, Phys. Rev. B 54, 11169 (1996).

${ }^{32}$ J. P. Perdew, K. Burke, and M. Ernzerhof, Phys. Rev. Lett. 77, 3865 (1996).

${ }^{33}$ J. D. Pack and H. J. Monkhorst, Phys. Rev. B 16, 1748 (1977).

${ }^{34}$ G. Kresse and D. Joubert, Phys. Rev. B 59, 1758 (1999).

${ }^{35}$ S. P. Russo, I. E. Grey, and N. C. Wilson, J. Phys. Chem. C 112, 7653 (2008).

${ }^{36}$ G. Kresse and J. Furthmuller, Comput. Mater. Sci. 6, 15 (1996).

${ }^{37}$ J. J. Rehr, J. J. Kas, F. D. Vila, M. P. Prange, and K. Jorissen, Phys. Chem. Chem. Phys. 12, 5503 (2010).

${ }^{38}$ J. T-Thienprasert, J. Nukeaw, A. Sungthong, S. Porntheeraphat, S. Singkarat, D. Onkaw, S. Rujirawat, and S. Limpijumnong, Appl. Phys. Lett. 93, 051903 (2008).

${ }^{39}$ O. Bunău and Y. Joly, J. Phys.: Condens. Matter 21, 345501 (2009).

${ }^{40}$ L. Romano, G. Impellizzeri, and M. G. Grimaldi, Mater. Sci. Semicond. Process. 15, 703 (2012).

${ }^{41}$ K. Bjoürkqvist, Appl. Phys. Lett. 13, 379 (1968).

${ }^{42}$ K. Björkqvist, D. Sigurd, G. Fladda, and G. Bjarnholt, Radiat. Eff. 6, 141 (1970). 